\title{
Review of: "External validation of the 4C mortality score among COVID-19 patients admitted to hospital in Ontario, Canada: a retrospective study"
}

Fernando Anschau ${ }^{1}$

1 Pontifícia Universidade Católica do Rio Grande do Sul

Potential competing interests: The author(s) declared that no potential competing interests exist.

This is a retrospective study to validate a mortality score. The topic is clinically relevant and, in light of the SARS-Cov-2 pandemic, very current and pertinent. The study showed that the AUC of the 4C score was $0.77(95 \% \mathrm{Cl}, 0.79-0.87)$ and therefore appears to be a valid tool for prognosticating COVID-19 mortality in Canadian hospitals.

The methodology is well described and the results are presented synthetically and correctly. Because they use retrospective data, the authors could make the potential measurement biases clearer when addressing the limitations of the study. Although they refer to the retrospective data, I believe it would be interesting to clarify these points; likewise the classification of obesity only by the definition of staff (and not by a more detailed measurement of weight and height with body mass index calculation), so that we could identify the "weight" of these variables in the data set. 